\title{
A New Differential Quadrature Methodology Based on Bernstein Polynomials for Solving the Equations Governing the Unsteady Flow of a Polytropic Gas
}

\author{
Abdul-Sattar J. Al-Saif ${ }^{1}$ and Firas A. Al-Saadawi ${ }^{2}$ \\ 1. Department Mathematics, Education College for pure Science, Basrah University, Basrah, Iraq \\ 2. Directorate of Education in Basrah, Basrah, Iraq
}

\begin{abstract}
Aims of this paper are to improve ADI differential quadrature method (ADI-DQM) based on Bernstein polynomials and add a new application to the differential quadrature method. By using the new methodology, the numerical solutions of the governing equations of unsteady two-dimensional flow of a polytropic gas are investigated. The numerical results reveal that the new technique is very effective and gives high accuracy, good convergence and reasonable stability.
\end{abstract}

Key words: Differential quadrature method, polytropic gas, Bernstein polynomial, ADI, accuracy.

\section{Introduction}

Nonlinear phenomena attracted the attention scientific community due to its widely applications in sciences and engineering such as solid state physics, plasma physics, fluid dynamics, mathematical biology, chemical kinetics, etc. These applications are description by modelling of differential equations. The studies conducted for solving the equation governing the unsteady flow of a polytropic gas use a broad class of analytical methods and different types of numerical methods were used to handle such problems [1-7].

The differential quadrature method is a powerful mathematical technique and very useful to find a numerical solutions of the nonlinear equations and considered one of the most important of the numerical methods used recently, because it used less number of the grid points comparison with other numerical methods as well as the accuracy and efficiency. This method was suggested by Bellman and Casti in the

Corresponding author: Abdul-Sattar Jaber Ali Al-Saif, $\mathrm{Ph} . \mathrm{D}$, assistant professor, research fields: applied mathematics (numerical analysis, partial differential equations, dynamics system, computational of fluid mechanics). E-mail: sattaralsaif@yahoo.com. early 1970s [8]. Differential quadrature method (DQM) depends on the idea of integral quadrature and approximate a spatial partial derivatives as a linear weighted sum of all functional values of the solution at all mesh points [9]. One of most important keys to DQM lies in the determination of weighting coefficients for the discretization of a spatial derivatives of any order, where it plays the important role in the accuracy of numerical solutions. Bellman et al. [10] (1972) suggested two methods to determine the weighting coefficients of the first order derivative. The first method solves an algebraic equation system. The second use a simple algebraic formulation, but with the coordinates of grid points chosen as the roots of the Legendre polynomial. Quan and Chang [11] (1989) and Shu and Richards [12] (1992), derived a recursive formula to obtain these coefficients directly and irrespective of the number and positions of the sampling points. In their approach, they used the Lagrange polynomials as the trial functions and found a simple recurrence formula for the weighting coefficients. Bert et al. [13] (1993) and Striz et al. [14] (1995) developed the differential quadrature method, which uses harmonic functions instead of polynomial 
as test function in the quadrature method to handle periodic problems efficiently, and also circumvented the limitation for the number of grid point in the conventional DQM based on polynomial test function. Their study shows that the proper test functions are essential for the computational efficiency and reliability of the DQM Krowiak [15] (2008) studied the methods that based on the differential quadrature in vibration analysis of plates, and using the spline functions as the trial functions to compute weighting coefficients. Shu and Wu [16] in (2007), presented integrated radial basis functions based differential quadrature method and its performance for solving one-dimensional Burger equation and simulate natural convection in a concentric annulus by solving Navier-Stokes equations by using radial basis functions as the trial functions to compute weighting coefficients. Korkmaz and Dağ [17] in (2009) applied the differential quadrature method based on Chebyshev polynomials for space discretiztion and classical Runge-Kutta method of the 4th-order for time discretization to obtain numerical solution of Schrödinger equation for various initial conditions. Korkmaz et al. [18] (2011) used the quartic B-spline differential quadrature method, and applied it on the one-dimensional Burger's equation by using the quartic B-spline functions as the trial functions to compute weighting coefficients. Al-Saif and Al-Kanani [19, 20] (2012-2013) proposed a new improvement for DQM that is resulting from applied ADI into DQM for convection-diffusion problems, and the results of ADI-DQM with Lagrange polynomial and Fourier series expansion as the test functions to computing the weighted coefficients show the efficiency of the proposed method to handle the problems under consideration.

We notice, attention all the above authors' to develop the DQM by using different test functions (Legendre polynomial, Chebyshev polynomials, Lagrange polynomials, harmonic functions instead of polynomials, Fourier series expansion, spline function, quartic B-spline functions and radial basis functions) in computing weighted coefficients of DQM because it plays an important role in an accurate numerical solutions that motivate us to research about polynomial has good properties and suitable with application of DQM. Bernstein polynomials are incredibly useful mathematical tools as they are simply defined. They can be calculated quickly on computer systems and represent a tremendous variety of functions. They can be differentiated and integrated easily, and can be pieced together to form spline curves that can approximate any function to any accuracy desired. One of important properties to Bernstein polynomials is surely convergence, and used many researchers to solve differential equations $[9,21,22]$.

In this paper, we wanted extending the application of our new suggestion [9], and improved ADI-DQM by using Bernstein polynomials for solving the equation governing the unsteady flow of a polytropic gas. Results show that the convergence of the new scheme is faster and the solutions have high accuracy, good convergence and reasonable stability.

\section{Bernstein Differential Quadrature Method}

The differential quadrature is a numerical technique used to solve the initial and boundary value problems. This method was proposed by Bellman and Casti [8] in 1971. The DQM is based on the idea that the partial derivative of a field variable at the $i^{\text {th }}$ discrete points in the computational domain is approximated by a weighted linear sum of the values of the field variable along the line that passes though that point, which is parallel with coordinate direction of the derivative as following [19, 20]:

$$
\begin{gathered}
\left.\frac{\partial^{m} u}{\partial x^{m}}\right|_{\substack{x=x_{i}, y=y_{j}}}=\sum_{k=1}^{N} w_{i k}^{(m)} u\left(x_{k}, y_{j}\right), i=1,2, \ldots, N, j \\
\quad=1, \ldots, M, m=1, \ldots, N-1 \\
\begin{aligned}
&\left.\frac{\partial^{n} u}{\partial y^{n}}\right|_{\substack{x=x_{i}, y=y_{j}}} \sum_{l=1}^{M} w_{j l}^{(n)} u\left(x_{i}, y_{l}\right), i=1,2, \ldots, N, j= \\
& 1, \ldots, M, n=1, \ldots, M-1
\end{aligned}
\end{gathered}
$$

where, $\left(x_{i}, y_{j}\right)$ are the discrete points in the variable, 
$u_{x}^{(m)}, u_{y}^{(n)}$ is the $m^{t h}$ and $n^{t h}$ order derivative of the function, $u\left(x_{i}, y_{j}\right)$ are the function values at these points, and $w_{i k}^{(m)}, w_{j l}^{(n)}$ are the weighting coefficients for the $m^{\text {th }}$ and $n^{\text {th }}$ order derivative of the function with respect to $x$ and $y$, and $N$ and $M$ are the number of the grid points. There are two key points in the successful application of the DQM: how the weighting coefficients are determined and how the grid points are selected [23]. Many researchers have obtained weighting coefficients implicitly or explicitly using various test functions [6, 9, 10, 12-20].

Here, we use the exact same manner in $\{$ Quan and Chang [11] (1989) and Shu and Richards [12] (1992)\} to determine the weighting coefficients, but with employing the Bernstein polynomials as the test function. A Bernstein polynomial, named after Sergei Natanovich Bernstein, is a polynomial in the Bernstein form, that is a linear combination of Bernstein basis polynomials. The Bernstein basis polynomials of $n^{\text {th }}$-degree are defined on the interval $[0,1]$ by Singh et al. [24]:

$$
B_{k, n}(x)=\left(\begin{array}{l}
n \\
k
\end{array}\right) x^{k}(1-x)^{n-k}, 0 \leq k \leq n
$$

The general form of Bernstein polynomials of $n^{\text {th }}$-degree that used to solve differential equation [21, $22]$ is defined on the interval $[a, b]$ as:

$$
B_{k, n}(x)=\left(\begin{array}{l}
n \\
k
\end{array}\right) \frac{(x-a)^{k}(b-x)^{n-k}}{(b-a)^{n}}, 0 \leq k \leq n
$$

where, binomial coefficients are given by :

$$
\left(\begin{array}{l}
n \\
k
\end{array}\right)=\frac{n !}{k !(n-k) !}
$$

There are $n+1, n^{t h}$-degree Bernstein polynomials. For mathematical convenience, we usually set $B_{k, n}(x)=0$, if $k<0$ or $k>n$. These polynomials are quite easy to write down the coefficients that can be obtained from Pascal's triangle. It can easily be shown that each of the Bernstein polynomials is positive and also the sum of all the Bernstein polynomials is unity for all real $x \in[a, b]$, i.e.:

$$
\sum_{k=0}^{n} B_{k, n}(x)=1, \quad \forall x \in[a, b]
$$

The Bernstein polynomials can be written to any interval as following [23]:

$$
b(x) \cong B_{n}(f)(x)=\sum_{k=0}^{n} f\left(\frac{k}{n}\right) B_{k, n}(x)
$$

where, $f\left(\frac{k}{n}\right)$ is arbitrary function, for $k=$ $0,1, \ldots, n, n \geq 1$. Similar to Lagrange differential quadrature method (LDQM) to determined weighting coefficients, we can derive the explicit formulation to compute the weighting coefficients $w_{i k}^{(1)}$ by using Bernstein polynomial as a test functions, which are listed below:

$$
w_{i k}^{(1)}=\left\{\begin{array}{c}
\frac{1}{L} \frac{b^{(1)}\left(x_{i}\right)}{\left(x_{i}-x_{k}\right) b^{(1)}\left(x_{k}\right)} \text { for } i \neq k, \\
\quad-\sum_{k=1, i \neq k}^{N} w_{i k}^{(1)}, \text { for } i=k \\
i, k=1,2, \ldots, N
\end{array}\right.
$$

where, $L=b-a$ is length interval $[a, b]$ and

$$
b(x)=\sum_{k=0}^{n} f\left(\frac{k}{n}\right)\left(\begin{array}{l}
n \\
k
\end{array}\right) \frac{(x-a)^{k}(b-x)^{n-1}}{(b-a)^{n}}
$$

and

$$
b^{(1)}\left(x_{i}\right)=\sum_{k=0}^{n} f\left(\frac{k}{n}\right)\left(\begin{array}{l}
n \\
k
\end{array}\right) \frac{\left(x_{i}-a\right)^{k}\left(b-x_{i}\right)^{n-1}}{(b-a)^{n}}
$$

The weighted coefficients of the second order derivative by using Bernstein polynomial as a test functions can be obtained:

$$
\left[w_{i k}^{(2)}\right]=\left[w_{i k}^{(1)}\right]\left[w_{i k}^{(1)}\right]=\left[w_{i k}^{(1)}\right]^{2}
$$

The same technique can be used to obtain the weighting coefficients $w_{i k}^{(m)}$.

\section{Alternating Direction Implicit Technique-BDQM}

To illustrate the application of the technique of ADI to the formulae of DQM, we consider the following partial differential equation in two dimensions as:

$$
\frac{\partial u}{\partial t}=\ell_{x} u+\ell_{x} p+\ell_{y} u
$$

where, $\ell_{x}$ and $\ell_{y}$ are the differential operators with respect to $x$ and $y$, respectively.

The alternating direction implicit technique was 
introduced in the middle 1950s by Peaceman and Rachford for solving equations, which result from finite difference discretization of partial differential equations (PDEs). From iterative method's perspective, ADI method can be considered as special relaxation method, where a big system is simplified into a number of smaller systems such that each of them can be solved efficiently and the solution of the whole system is got from the solutions of the sub-systems in an iterative way. Using alternating direction implicit method to approximate Eq. (8), we get the systems of algebraic equations in the form [19, 20]:

$$
\begin{gathered}
\frac{u_{i j}^{n+\frac{1}{2}}-u_{i j}^{n}}{\frac{\Delta t}{2}}=\sum_{k=1}^{N}\left(\left(w_{d q x}\right)_{i k}\right) u_{i k}^{n+\frac{1}{2}} \\
+\sum_{k=1}^{N}\left(\left(w_{d q x}\right)_{i k}\right) p_{i k}^{n+\frac{1}{2}}+\sum_{l=1}^{M}\left(\left(w_{d q y}\right)_{j l}\right) u_{j l}^{n} \\
\frac{u_{i j}^{n+1}-u_{i j}^{n+\frac{1}{2}}}{\frac{\Delta t}{2}}=\sum_{k=1}^{N}\left(\left(w_{d q x}\right)_{i k}\right) u_{i k}^{n+\frac{1}{2}} \\
+\sum_{k=1}^{N}\left(\left(w_{d q x}\right)_{i k}\right) p_{i k}^{n+\frac{1}{2}}+\sum_{l=1}^{M}\left(\left(w_{d q y}\right)_{j l}\right) u_{j l}^{n+1}
\end{gathered}
$$

where, $\left(w_{d q x}\right)_{i k}$ and $\left(w_{d q y}\right)_{j l}$ are the BDQM quantities that are including the weighting coefficients for the differential operators $\ell_{x}$ and $\ell_{y}$, respectively.

Eq. (9) is used to compute function values at all interval mesh points along rows and known as horizontal traverse or $x$-sweep. While, Eq. (10) is used to compute function values at all interval mesh points along columns and known as vertical traverse or $y$-sweep.

\section{Application of BDQM and ADI-BDQM and Discussion}

Consider the governing equations of unsteady two dimensional flow of a polytropic gas are given by Feng [2], Sharma et al. [6], Mohamed [5] and Matinfar et al. [4]:

$$
\begin{gathered}
u_{t}+u u_{x}+v u_{y}+\frac{p_{x}}{\rho}=0 \\
v_{t}+u v_{x}+v v_{y}+\frac{p_{y}}{\rho}=0 \\
\rho_{t}+u \rho_{x}+v \rho_{y}+\rho\left(u_{x}+v_{y}\right)=0 \\
p_{t}+u p_{x}+v p_{y}+\gamma p\left(u_{x}+v_{y}\right)=0
\end{gathered}
$$

where, $\rho$ is the density, $p$ is the pressure, $u$ and $v$ are the velocity components in the $x$ and $y$ direction, respectively and the adiabatic index $\gamma$ is the ratio of the specific heats.

The initial conditions of Eqs. (11)-(14) have the following forms:

$$
\left.\begin{array}{c}
u(x, y, 0)=e^{x+y}, v(x, y, 0)=-1-e^{x+y} \\
\rho(x, y, 0)=e^{x+y}, p(x, y, 0)=\mathrm{c}
\end{array}\right\}
$$

The exact solutions of the equations are

$$
\left.\begin{array}{c}
u(x, y, t)=e^{x+y+t}, v(x, y, t)=-1-e^{x+y+t} \\
\rho(x, y, t)=e^{x+y+t}, p(x, y, t)=\mathrm{c}
\end{array}\right\}
$$

where, $\mathrm{c}$ is constant. The boundary conditions can be achieved easily from Eq. (16) by using $x, y=0,1$. Eq. (11) can be approximated by using BDQM, form Eqs. (1a), (1b) and (6) as following:

$$
\begin{gathered}
u_{i j}^{n+1}=u_{i j}^{n}-\Delta t\left[\sum_{k=1}^{N} u_{i j}^{n} w_{i k}^{(1)} u_{k j}^{n}\right. \\
+\sum_{k=1}^{N} v u_{i j}^{n} \widetilde{w}_{j l}^{(1)} u_{i l}^{n}+\sum_{k=1}^{N} u_{i j}^{n} w_{i k}^{(1)} u_{k j}^{(1)}
\end{gathered}
$$

Eq. (11) can be approximated by using ADI-BDQM, form Eqs. (1a), (1b), (6), (9) and (10), such that $\left(w_{d q x}\right)_{i k}=u_{i j}^{n} w_{i k}^{(1)},\left(w_{d q y}\right)_{j l}=v_{i j}^{n} w_{j l}^{(1)}$ of $u$ and $\left(w_{d q x}\right)_{i k}=\frac{1}{\rho_{i j}^{n}} w_{i k}^{(1)}$ of $p$ as following:

$$
\begin{gathered}
u_{i j}^{n+\frac{1}{2}}=u_{i j}^{n}-\frac{\Delta t}{2}\left[\sum_{k=1}^{N} u_{i j}^{n} w_{i k}^{(1)} u_{i k}^{n+\frac{1}{2}}\right. \\
\left.+\sum_{k=1}^{N} \frac{1}{\rho_{i j}^{n}} w_{i k}^{(1)} p_{k j}^{n+\frac{1}{2}}+\sum_{l=1}^{M} v_{i j}^{n} w_{j l}^{(1)} u_{j l}^{n}\right] \\
u_{i j}^{n+1}=u_{i j}^{n+\frac{1}{2}}-\frac{\Delta t}{2}\left[\sum_{k=1}^{N} u_{i j}^{n+\frac{1}{2}} w_{i k}^{(1)} u_{i k}^{n+\frac{1}{2}}\right. \\
\left.+\sum_{k=1}^{N} \frac{1}{\rho_{i j}^{n+\frac{1}{2}}} w_{i k}^{(1)} p_{k j}^{n+\frac{1}{2}}+\sum_{l=1}^{M} v_{i j}^{n+\frac{1}{2}} w_{j l}^{(1)} u_{j l}^{n+1}\right]
\end{gathered}
$$

where, the $w_{i k}^{(1)}$ and $w_{j l}^{(1)}$ are the weighting 


\section{A New Differential Quadrature Methodology based on Bernstein Polynomials for Solving the Equations Governing the Unsteady Flow of a Polytropic Gas}

coefficients of the first order derivatives with respect to $x$ and $y$, respectively. In the same technique, we can find numerical solutions for $v$ and $\rho$ by using BDQM and ADI-BDQM.

In this problem, we take $L=1, c=4, \gamma=-2$, and use equally spaced grid points. Tables 1-3 show the errors obtained from solving the equations governing the unsteady flow of a polytropic gas by using LDQM, BDQM, ADI-LDQM and ADI-BDQM at $t=0.01, \Delta t=$ 0.001 and $x$ and $y \in[0,1]$ for different values of $h$ $=\Delta x=\Delta y$. Figs. 1-3 clarify a comparison between exact solution and numerical solutions of the gas problem with respect to $L=3$. The results show that the ADI-BDQM and BDQM have a high accuracy, good convergence and less CPU time compared with the LDQM and ADI-LDQM.

We choose the arbitrary function $f\left(\frac{k}{n}\right)=$ $\exp \left(s \cdot \frac{k}{n} \cdot h\right), \quad k=0,1, \ldots, n$ and $s$ is the arbitrary constant. In this problem, we take $s=-20,47,107$ and 457.

\section{Errors Analysis}

The error for the $m^{\text {th }}-$ ord derivative approximation an be written as:

$$
\varepsilon_{D}^{(m)}\left(x_{i}\right)=\frac{\partial^{(m)}\left(u^{(N)}(\xi) \cdot b\left(x_{i}\right)\right)}{(N) ! \partial x^{m}}
$$

Bellman et al. (1972) are using the error resulting from approximation functions and its derivatives. The truncation error of a new methodology BDQM for the 1 st- and the 2 nd order derivatives at the grid point is given as:

$$
\begin{gathered}
\varepsilon^{(1)}\left(x_{i}\right) \leq \frac{K_{1} b^{(1)}\left(x_{i}\right)}{N !}=K_{1} \cdot e_{1}\left(x_{i}\right), \\
i=1,2, \ldots, N
\end{gathered}
$$

where, $K_{1}=\operatorname{Max}\left\{\left|u^{(N)}(\xi)\right|\right\}$,

$$
\begin{gathered}
b^{(1)}\left(x_{i}\right)=\sum_{k=0}^{n} f\left(\frac{k}{n}\right) B_{k, n}^{(1)}\left(x_{i}\right) \\
\varepsilon^{(2)}\left(x_{i}\right) \leq 2 K_{2}\left(1+w_{i i}^{(1)}\right) \frac{b^{(2)}\left(x_{i}\right)}{N !}=K_{2} \cdot e_{2}\left(x_{i}\right), i \\
=1,2, \ldots, N
\end{gathered}
$$

where,

$$
\begin{aligned}
& K_{1}= \operatorname{Max}\left\{\left|u^{(N)}(\xi)\right|,\left|\xi_{x} u^{(N+1)}(\xi)\right|\right\}, b^{(2)}\left(x_{i}\right)= \\
& \sum_{k=0}^{n} f\left(\frac{k}{n}\right) B_{k, n}^{(2)}\left(x_{i}\right) \\
& \quad x_{i}\left(1-x_{i}\right) B_{k, n}^{(1)}\left(x_{i}\right)=\left(k-n x_{i}\right) B_{k, n}\left(x_{i}\right)
\end{aligned}
$$

where,

Table 1 Errors obtained for gas problem with $t=0.01$ of $u$.

\begin{tabular}{lllllllll}
\hline$h$ & $\begin{array}{l}\text { Max|error| } \\
\text { LDQM }\end{array}$ & CPU & $\begin{array}{l}\text { Max|error| } \\
\text { ADI-LDQM }\end{array}$ & CPU & $\begin{array}{l}\text { Max |error| } \\
\text { BDQM }\end{array}$ & CPU & $\begin{array}{l}\text { Max|error| } \\
\text { ADI-BDQM }\end{array}$ & CPU \\
\hline 0.250 & $3.370728 \mathrm{E}-04$ & 0.004 & $2.736206 \mathrm{E}-04$ & 0.005 & $2.091217 \mathrm{E}-04$ & 0.003 & $5.486291 \mathrm{E}-05$ & 0.004 \\
0.170 & $2.701140 \mathrm{E}-04$ & 0.005 & $1.938115 \mathrm{E}-04$ & 0.006 & $1.106625 \mathrm{E}-04$ & 0.004 & $3.239892 \mathrm{E}-05$ & 0.005 \\
0.125 & $2.441108 \mathrm{E}-04$ & 0.008 & $1.621137 \mathrm{E}-04$ & 0.009 & $5.250823 \mathrm{E}-05$ & 0.005 & $2.390709 \mathrm{E}-05$ & 0.008 \\
0.100 & $2.304611 \mathrm{E}-04$ & 0.011 & $1.452148 \mathrm{E}-04$ & 0.013 & $2.077072 \mathrm{E}-05$ & 0.008 & $2.851537 \mathrm{E}-06$ & 0.012 \\
\hline
\end{tabular}

Table 2 Errors obtained for gas problem with $t=0.01$ of $v$.

\begin{tabular}{lllllllll}
\hline$h$ & $\begin{array}{l}\text { Max|error| } \\
\text { LDQM }\end{array}$ & CPU & $\begin{array}{l}\text { Max|error| } \\
\text { ADI-LDQM }\end{array}$ & CPU & $\begin{array}{l}\text { Max|error| } \\
\text { BDQM }\end{array}$ & CPU & $\begin{array}{l}\text { Max|error| } \\
\text { ADI-BDQM }\end{array}$ & CPU \\
\hline 0.250 & 3.732997E-04 & 0.004 & $3.384969 \mathrm{E}-04$ & 0.004 & $3.096577 \mathrm{E}-05$ & 0.003 & $8.294955 \mathrm{E}-06$ & 0.003 \\
0.170 & $2.972357 \mathrm{E}-04$ & 0.005 & $2.537857 \mathrm{E}-04$ & 0.005 & $2.949248 \mathrm{E}-05$ & 0.004 & $9.643521 \mathrm{E}-06$ & 0.004 \\
0.125 & $2.658443 \mathrm{E}-04$ & 0.008 & $2.184850 \mathrm{E}-04$ & 0.009 & $8.644646 \mathrm{E}-06$ & 0.005 & $1.255671 \mathrm{E}-06$ & 0.008 \\
0.100 & $2.488420 \mathrm{E}-04$ & 0.010 & $1.992359 \mathrm{E}-04$ & 0.013 & $7.496638 \mathrm{E}-07$ & 0.008 & $2.093294 \mathrm{E}-07$ & 0.012 \\
\hline
\end{tabular}

Table 3 Errors obtained for gas problem with $t=0.01$ of $\rho$.

\begin{tabular}{lllllllll}
\hline$h$ & $\begin{array}{l}\text { Max|error| } \\
\text { LDQM }\end{array}$ & CPU & $\begin{array}{l}\text { Max|error| } \\
\text { ADI-LDQM }\end{array}$ & CPU & $\begin{array}{l}\text { Max |error| } \\
\text { BDQM }\end{array}$ & CPU & $\begin{array}{l}\text { Max|error| } \\
\text { ADI-BDQM }\end{array}$ & CPU \\
\hline 0.250 & $4.049881 \mathrm{E}-04$ & 0.003 & $3.534122 \mathrm{E}-05$ & 0.004 & $7.324002 \mathrm{E}-06$ & 0.002 & $5.153906 \mathrm{E}-06$ & 0.003 \\
0.170 & $3.519990 \mathrm{E}-04$ & 0.005 & $3.214508 \mathrm{E}-04$ & 0.005 & $3.034167 \mathrm{E}-06$ & 0.003 & $2.176259 \mathrm{E}-06$ & 0.004 \\
0.125 & $3.334495 \mathrm{E}-04$ & 0.007 & $3.491768 \mathrm{E}-04$ & 0.009 & $8.617753 \mathrm{E}-05$ & 0.005 & $7.029928 \mathrm{E}-05$ & 0.008 \\
0.100 & $3.245713 \mathrm{E}-04$ & 0.011 & $2.633765 \mathrm{E}-04$ & 0.013 & $2.730333 \mathrm{E}-04$ & 0.007 & $1.691770 \mathrm{E}-04$ & 0.012 \\
\hline
\end{tabular}



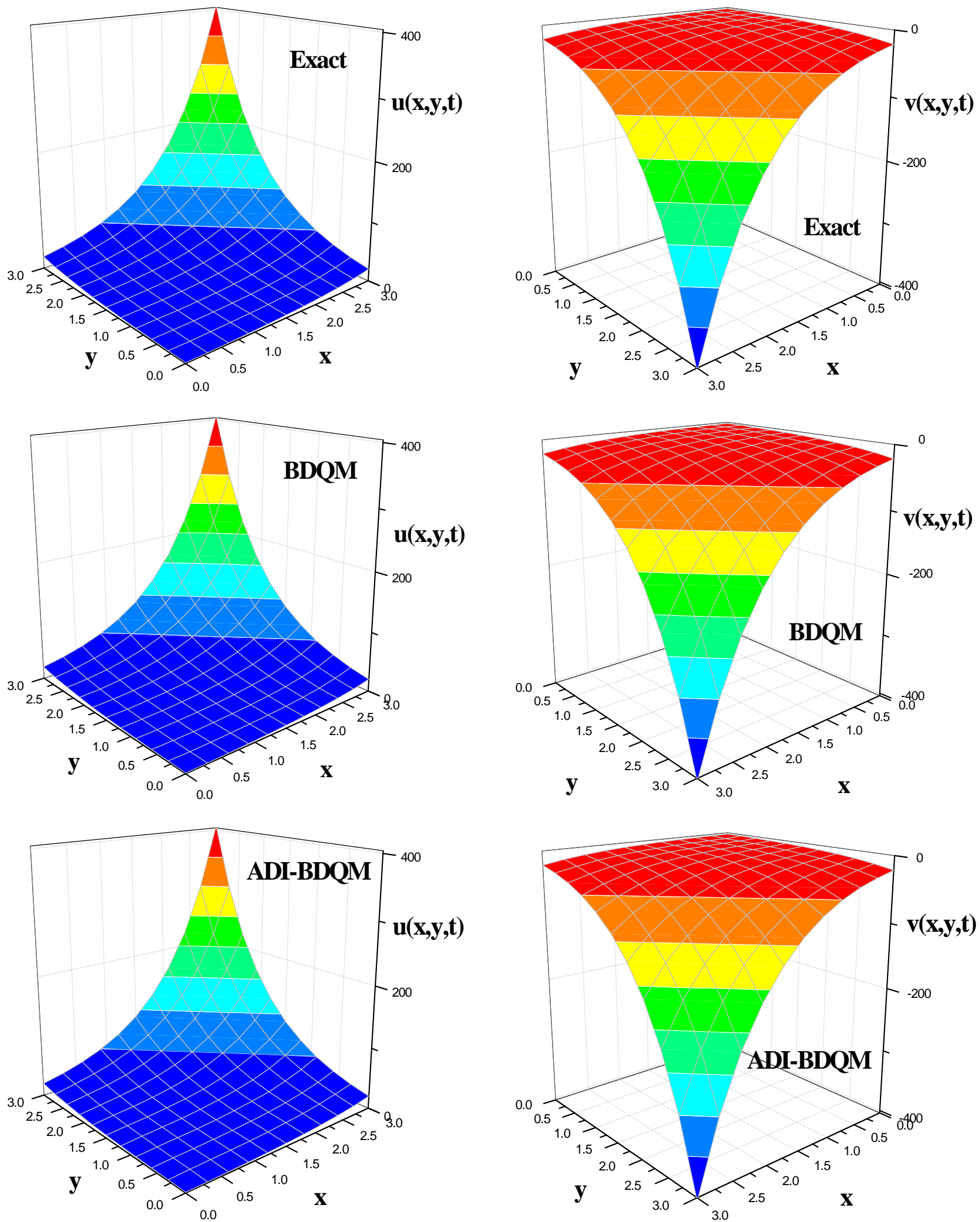

Fig. 1 Exact and approximate solutions of gas problem with, $t=0.01$.

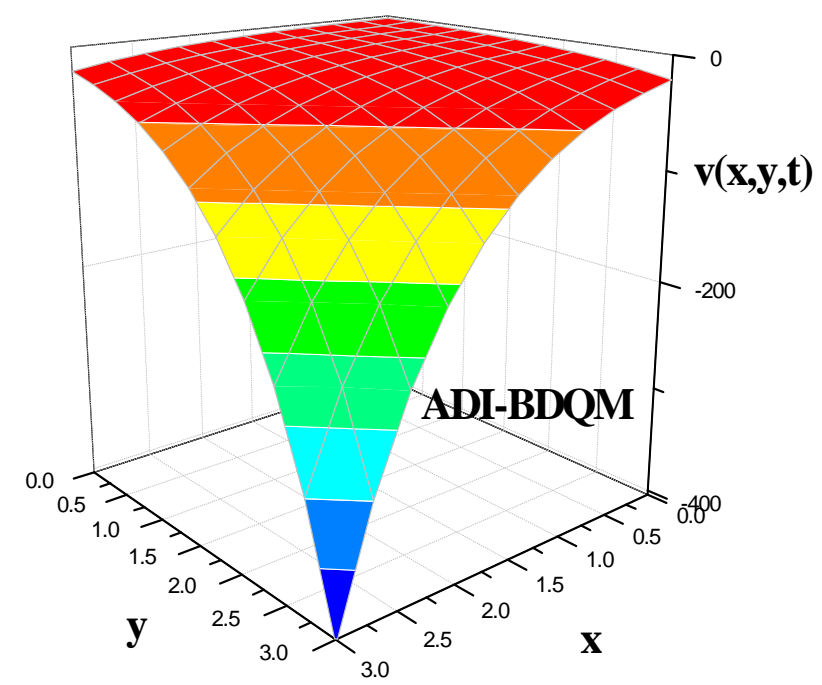

Fig. 2 Exact and approximate solutions of gas problem with, $t=0.01$. 

Governing the Unsteady Flow of a Polytropic Gas
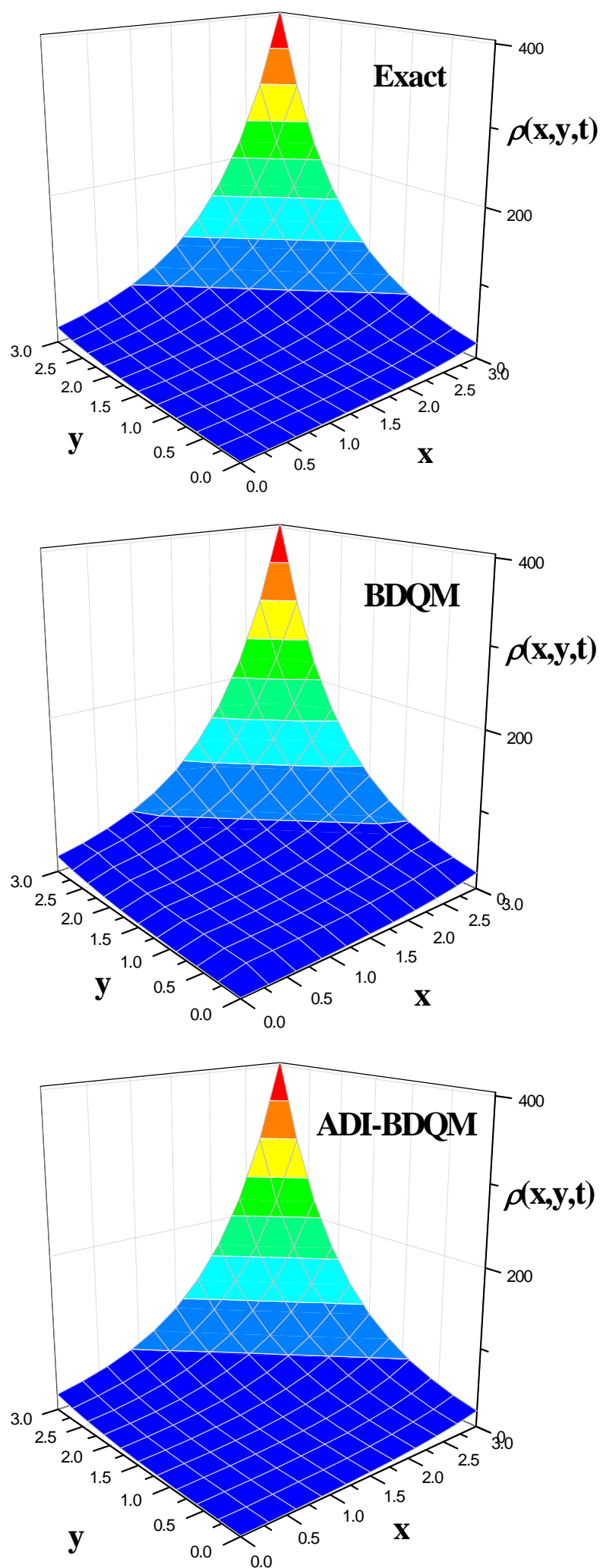

Fig. 3 Exact and approximate solutions of gas problem with, $t=0.01$.

$$
\begin{aligned}
& x_{i}\left(1-x_{i}\right) \leq \frac{1}{4} \\
& \begin{aligned}
\left(x_{i}\left(1-x_{i}\right)\right)^{2} B_{k, n}^{(2)}\left(x_{i}\right) \\
\quad=\left(\left(k-n x_{i}\right)^{2}+2 k x_{i}-n x_{i}^{2}\right. \\
\quad-k) B_{k, n}\left(x_{i}\right)
\end{aligned}
\end{aligned}
$$

where, $\left(x_{i}\left(1-x_{i}\right)\right)^{2} \leq \frac{1}{16}$.

\section{Stability Analysis}

The stability of numerical schemes is closely related to numerical error. A solution is said to be unstable if errors appear at some stages in the calculations (for example, from erroneous initial conditions or local truncation or round-off errors) are propagated without bound throughout subsequent calculations. Thus a method is stable if small changes in the initial data produce correspondingly small changes in the final results, that is, the difference between the theoretical and numerical solutions remains bounded at a given time $t$, as time and space steps tend to zero or time step remains fixed at every level and $t \rightarrow \infty$ [19]. So stability, means that the numerical solution must be close to the exact solution, meaning that whenever was the error a little the deviation in derivatives, however, this error may accumulate at each time step and affects to the stability of the solution.

Theorem 1 [25]: the system of ODE $\hat{u}=\mathrm{A} u$ with a coefficient matrix constant $\mathrm{A}$ is:

(1) Stable if the roots of the characteristic polynomial are purely imaginary;

(2) Asymptotically stable if the roots have negative real

Parts;

(3) Unstable if a root has positive real part.

From application of BDQM to the any partial differential equation, we obtained the set of ordinary differential equations:

$$
[A]\{u\}=\{b\}-\{r\}
$$

where, $\{u\}$ is a vector of unknown functional values at all the interior points given by: 


\section{A New Differential Quadrature Methodology based on Bernstein Polynomials for Solving the Equations Governing the Unsteady Flow of a Polytropic Gas}

$$
\begin{array}{r}
\{u\}=\left[u_{2,2}, u_{2,3}, \ldots, u_{2, M-1}, u_{3,2}, \ldots,\right. \\
\left.u_{3, M-1}, \ldots, u_{N-1,2}, \ldots, u_{N-1, M-1}\right]^{T}
\end{array}
$$

$\{b\}$ is a vector still containing discretized time derivatives of $u$ and $\{r\}$ is a known vector which is made up of the non-homogeneous part and the boundary conditions given by:

$$
\begin{aligned}
& \{r\}=\left[r_{2,2}, r_{2,3}, \ldots, r_{2, M-1}, r_{3,2}, \ldots\right. \\
& \left.r_{3, M-1}, \ldots, r_{N-1,2}, \ldots, u_{N-1, M-1}\right]^{T}
\end{aligned}
$$

and $[A]$ is the coefficient matrix containing the weighting coefficients, the dimension of the matrix $[A]$ is $(N-2)(M-2)$ by $(N-2)(M-2)$. For the multi-dimensional case, the matrix $[A]$ contains many zero elements, which are irregularly distributed in the matrix.

The stability analysis of the Eq. (23) is based on the eigenvalue distribution of the BDQM discretization matrix $[A]$. If $[A]$ has eigenvalues $\lambda_{i}$ and corresponding eigenvectors $\xi_{i}(i=1,2, \ldots K) K$ being the size of the matrix $[A]$, the similarity transformation reduces the system (23) of the form [12].

$$
\frac{\mathrm{d}\{U\}}{\mathrm{d} t}=[D]\{U\}+\{R\}
$$

here, the diagonal matrix $[D]$ is formed from the eigenvalues and from a nonsingular matrix $[P]$ containing the eigenvectors as columns:

$$
[D]=[P]^{-1}[A][P]
$$

Pre-multiplying by the matrix $[P]^{-1}$ on the both sides of Eq. (5.36) and setting:

$$
\begin{aligned}
\{U\} & =[P]^{-1}[u] \\
\{R\} & =[P]^{-1}[r]
\end{aligned}
$$

Since $[D]$ is a diagonal matrix, Eq. (24) is an uncoupled set of ordinary differential equations. Considering the $i^{\text {th }}$ in Eq. (24):

$$
\frac{\mathrm{d} U_{i}}{\mathrm{~d} t}=\lambda_{i} U_{i}+R_{i}
$$

The solution of Eq. (28) consists of two parts: the complementary solution and the particular solution. If $R_{i}$ is time-independent, then the solution of Eq. (28) can be written as:

$$
U_{i}=\left(U_{i}(0)+\frac{R_{i}}{\lambda_{i}}\right) e^{\lambda_{i} t}-\frac{R_{i}}{\lambda_{i}}
$$

For this case, using Eqs. (26) and (27), the solution $\{u\}$ can be obtained as:

$$
\begin{gathered}
\{u\}=[P][U]=\sum_{i=1}^{N} U_{i} \xi_{i} \\
=\sum_{\mathrm{i}=1}^{N}\left[U_{I}(0) e^{\lambda_{i} t}+\frac{R_{i}}{\lambda_{\mathrm{i}}}\left(e^{\lambda_{i} t}-1\right)\right] \xi_{\mathrm{i}}
\end{gathered}
$$

Clearly, the stable solution of $\{u\}$ when $t \rightarrow$ $\infty$ requires:

$$
\text { Real }\left(\lambda_{i}\right) \leq 0 \text { for all } i
$$

where, Real $\left(\lambda_{i}\right)$ denotes the real part of $\lambda_{i}$. This is the stability condition for Eq. (23). Now, we can apply the stability Eq. (31) on the gas problem. From the application of BDQM and ADI-BDQM to the Eq. (1) and using $N=M=5$, we have obtained good results in the measure of stability (Table 4). That means the stability Eq. (31) is hold.

When using the $v$-Eq. (12), we will find the same eigenvalues mentioned in Table 4 of the matrix $[A]$. In this work, we have used Maple program to compute the eigenvalues. In the multi-dimensional case, the dimension of the matrix $[A]$ is $(N-2)(M-2)$ by

Table 4 Comparison of the Real $\left(\lambda_{i}\right)$ of the gas problem/ $u$-equation.

\begin{tabular}{cllll}
\hline$N \times M$ & Real $\left(\lambda_{i}\right)$ & LDQM & BDQM & ADI-BDQM \\
\hline \multirow{6}{*}{$5 \times 5$} & $\lambda_{1}$ & -0.089820272 & -8.244439720 & -8.217430755 \\
& $\lambda_{2}$ & -0.089820272 & -8.244439720 & -8.217430755 \\
& $\lambda_{3}$ & -2.067156321 & -10.66512874 & -11.08554125 \\
& $\lambda_{4}$ & -2.067156321 & -10.66512874 & -11.08554125 \\
& $\lambda_{5}$ & -2.156976732 & -0.012753540 & -0.074360796 \\
& $\lambda_{6}$ & -2.156976732 & -0.012753540 & -0.074360796 \\
& $\lambda_{7}$ & 0.000000029 & -2.803609271 & -2.884787132 \\
& $\lambda_{8}$ & 0.000000165 & -2.803609271 & -2.884787132 \\
& $\lambda_{9}$ & 0.000000165 & -5.578517640 & -6.588010258 \\
\hline
\end{tabular}



Governing the Unsteady Flow of a Polytropic Gas

Table 5 Comparison of the numerical results for gas problem at $t=0.01$.

\begin{tabular}{lllll}
\hline Method & Grid points & Max $\mid$ error $\mid$ of $u$ & Max $\mid$ error $\mid$ of $v$ & Max $\mid$ error $\mid$ of $\rho$ \\
\hline ADI-BDQM & $5 \times 5$ & $5.486291 \mathrm{E}-05$ & $8.294955 \mathrm{E}-06$ & $5.153906 \mathrm{E}-06$ \\
BDQM & $5 \times 5$ & $2.091217 \mathrm{E}-04$ & $3.096577 \mathrm{E}-05$ & $7.324002 \mathrm{E}-06$ \\
LDQM & $5 \times 5$ & $2.736206 \mathrm{E}-04$ & $3.384969 \mathrm{E}-04$ & $4.049881 \mathrm{E}-04$ \\
ADI-LDQM & $5 \times 5$ & $3.370728 \mathrm{E}-04$ & $3.732997 \mathrm{E}-04$ & $3.534122 \mathrm{E}-05$ \\
\hline
\end{tabular}

$(N-2)(M-2)$. Finally, we see the newly developed methods (BDQM and ADI-BDQM) are stable for the grid points $N=M=5$, and the numerical results confirm this fact.

\section{Comparison Numerical Solutions}

We compare the numerical results of ADI-BDQM, ADI-LDQM, BDQM and LDQM for a polytropic gas problem, and the results are listed in Table 5. The error measurements resulted from the ADI-BDQM is more accurate than the other methods.

\section{Conclusions}

In this work, we employed a new differential quadrature methodology (BDQM and ADI-BDQM) based on Bernstein polynomials for solving a polytropic gas equation successfully. The numerical results show that the new methods have higher accuracy, good convergence, reasonable stability and less computation workload.

\section{References}

[1] Al-Saif, A. S. J., and Al-Saadawi, F. A. 2013. "Bernstein Differential Quadrature Method for Solving the Unsteady State Convection-Diffusion Equation." Indian J. Appl. Res. 3 (9): 20-6.

[2] Al-Saif, A. S. J., and Al-Kanani, M. J. 2013. "Solution of Nonlinear Initial-Value Problems by the Alternating Direction Implicit Formulation on Differential Quadrature Method." Int. J. of Pure and Appl. Research in Engin. and Technology 1 (9): 57-69.

[3] Al-Saif, A. S. J., and Al-Kanani, M. J. 2012. "Alternating Direction Implicit Formulation of the Differential Quadrature Method for Solving Burger Equations." Int. J. Mod. Math. Sc. 30 (1): 1-11.

[4] Bellman, R., and Casti, J. 1971. "Differential Quadrature and Long-Term Integration.” J. Math. Analy. Appl. 34: 235-8.

[5] Bellman, R., Kashef, B. G., and Casti, J. 1972.
"Differential Quadrature: A Technique for the Rapid Solution of Nonlinear Partial Differential Equations." $J$. Comput. Phys. 10: 40-52.

[6] Bert, C. W., Wang, X., and Striz, A. G. 1993. "Differential Quadrature for Static and Free Vibration Analysis of Anisotropic Plates." Int. J. Solids Structures 30: 1737-44.

[7] Shaer, M. E. 2003. "Exact Solution of Equations of Motion a Polytropic Gas." Appl. Math. Comput. 141: 597-609.

[8] Feng, X. 1996. "Exact Wave front Solutions to Two Generalized Coupled Nonlinear Physical Equations." Physics Letters A 213: 167-76.

[9] Korkmaz, A., Aksoy, A. M., and Dag, I. 2011. "Quartic B-Spline Differential Quadrature Method." Int. J. of Nonlinear Sc. 11: 403-11.

[10] Korkmaz, A., and Dağ, İ. 2009. “A Differential Quadrature Algorithm for Nonlinear Schrödinger Equation." Nonlinear Dyn 56: 69-83.

[11] Krowiak, A. 2008. "Methods Based on the Differential Quadrature in Vibration Analysis of Plates." J. Theor. Appl. Mech. 46 (1): 123-39.

[12] Mantinfar, M., and Nodeh, S. 2009. “Application of He's Variational Iteration Method for Solving the Equation Governing the Unsteady Flow of a Polytropic Gas." $J$. Math. Ext. 3 (2): 61-7.

[13] Matinfar, M., and Saeidy, M. 2010. "Homotopy Analysis Method for Solving the Equation Governing the Unsteady Flow of a Polytropic Gas." World Appl. Sc. J. 9 (9): 980-3.

[14] Moghaddam, B., and Aghili, A. 2012. "A Numerical Method for Soliving Linear Non-homogenous Fractional Ordinary Differential Equation." Appl. Math. \& Inf. Sc. 6 (3): 441-5.

[15] Mohamed, M. 2009. "Adomian Decomposition Method for Solving the Equation Governing the Unsteady Flow of a Polytropic Gas." Appl. Appl. Math. 4 (1): 52-61.

[16] Mozaffari, M., Atai, A. A., and Mostafa, N. 2009. "Large Deformation and Mechanics of Flexible Isotropic Membrane Ballooning in Three Dimensions by Differential Quadrature Method." J. Mech. Sc. Tech. 23: 2921-7.

[17] Ordokhani, Y., and Far, S. D. 2011. "Application of the Bernstein Polynomials for Solving the Nonlinear Fredholm Integro-Differential Equations.” J. Appl. Math. Bioinf. 1: 13-31. 
[18] Quan, J., and Chang, C. 1989. "New Insights in Solving Distributed System Equations by the Quadrature Methods-I." Comput. Chem. Engng. A 13: 779-88.

[19] Sanchez, D. A. 1968. Ordinary Differential Equations and Stability Theory: An Introduction. USA: W.H. Freeman and Company.

[20] Sharma, P. R., and Methi, G. 2010. "Homotopy Perturbation Method Approach for Solution of Equation to Unsteady Flow of a Polytropic Gas." J. Appl. Sc. Res. 6 (12): 2057-62.

[21] Shu, C., and Richards, B. 1992. "Application of Generalized Differential Quadrature to Solve Two-Dimensional Incompressible Navier-Stokes
Equations.” Int. J. Numer. Meth. In Fluids 15: 791-8.

[22] Shu, C., and Wu, Y. L. 2007. "Integrated Radial Basis Functions-Based Differential Quadrature Method and Its Performance.” Int. J. Numer. Meth. Fluids 53: 969-84.

[23] Zayed, E. M., and Zedan, H. A. 2001. "Autonomous Forms and Exact Solutions of Equations of Motion of Polytropic Gas." Int. J. Theor. Phy. 40 (6): 1183-96.

[24] Singh, A. K., Singh, V. K., and Singh, O. P. 2009. "The Bernstein Operational Matrix of Integration." Appl. Math. Sc. 3 (49) 2427-36.

[25] Striz, A. G., Wang, X., and Bert, C. W. 1995. "Harmonic Differential Method and Applications to Structural Components." Acta Mechanica 111: 85-94. 